\title{
INCIDENCE OF FOREIGN BODY IN THE UPPER DIGESTIVE TRACT: A RETROSPECTIVE STUDY
}

\author{
Subhasish Mukherjee ${ }^{1}$, Afajul Hoque 2 , Suvamoy Chakraborty ${ }^{3}$ \\ ${ }^{1}$ Associate Professor, Department ENT, Sikkim Manipal Institute of Medical Sciences. \\ ${ }^{2}$ Senior Resident, Department ENT, Sikkim Manipal Institute of Medical Sciences. \\ ${ }^{3}$ Professor \& HOD, Department ENT, Sikkim Manipal Institute of Medical Sciences.
}

\section{ABSTRACT}

Foreign body impaction in the upper digestive tract is not an infrequent emergency in every clinical setup. In the present retrospective study, we have tried to analyze the incidence of impacted foreign body in the upper digestive tract at our center. The most frequent presentation is history of foreign body ingestion or aspiration. They are chiefly produced by accidental swallowing of the foreign body in the extremes of age and deliberate attempts in case of psychiatric and criminal background subjects. Varied manifestations of dysphagia, drooling of saliva and retro- sternal pain is presented. Additional symptoms of vomiting and subjective sensation of breathlessness are present occasionally. Removal of foreign body at times is a challenge for the gastrointestinal endoscopist. If not intervened expeditiously, can lead to horrific outcome. In our review study, rigid endoscopy resolved the problem in majority of cases by removal of the foreign body or dislodgement of the impacted fleshy meat ball in to the stomach. We report a retrospective study of incident and types of impacted foreign body in the upper digestive tract. The study was carried out at a Tertiary Care Center at Gangtok, India, spread over 5 years. The review of the case details was tabulated and the incidence types and outcome was calculated to review the clinical condition and its management.

\section{KEYWORDS}

Foreign Body, Impacted, Esophagoscopy, Endoscope.

HOW TO CITE THIS ARTICLE: Subhasish Mukherjee, Afajul Hoque, Suvamoy Chakraborty. "Incidence of Foreign Body in the Upper Digestive Tract: A Retrospective Study." Journal of Evolution of Medical and Dental Sciences 2015; Vol. 4, Issue 102, December 21; Page: $16765-16768$, DOI: $10.14260 /$ jemds/2015/2512

\section{INTRODUCTION}

Upper digestive tract foreign body impaction may present as an emergency and if not treated immediately may lead to catastrophic outcome. At the other end of the spectrum it may present as an occult impaction with no obvious presenting symptom. The first case of removal of foreign body from the upper digestive tract dates back to 1896. But it was in 1930 that Chevalier Jackson pioneered the illuminated rigid endoscope.[1] In 1966, Bigler reported on a new technique using Foley catheter and in 1970s and the 1980s the flexible fiber optic instrument became an option. ${ }^{[2]}$ Impacted foreign bodies are amenable to treatment by per oral endoscopy and rarely may require a thoracic surgeon's intervention.

Radiological localization of the impacted foreign body is mandatory before decision making for removal.[3] Failure to locate an object on X-rays does not preclude its presence. Thus in patients with typical clinical presentations or with highly suspected foreign body ingestion, an endoscopic evaluation should be performed even with a normal finding on radiography. [4] Fortunately, most of the foreign bodies pass through the gastrointestinal tract harmlessly. However, $10 \%$ to $20 \%$ will require non-operative intervention and only less than $1 \%$ will require surgery. ${ }^{[5]}$ However, untreated impacted foreign body may cause serious complications like visceral erosion and perforation, tracheo-esophageal fistula, mediastinitis and abscess formation. ${ }^{[6]}$

Financial or Other, Competing Interest: None.

Submission 24-11-2015, Peer Review 25-11-2015,

Acceptance 16-11-2015, Published 21-12-2015.

Corresponding Author:

Dr. Subhasish Mukherjee,

Associate Professor, ENT

Sikkim Manipal Institute of Medical Sciences,

Tadong, $5^{\text {th }}$ Mile, Gangtok-737102,

Sikkim.

E-mail:mukho162000@gmail.com

DOI:10.14260/jemds/2015/2512
Aspirated or ingested foreign bodies in the upper digestive tract often presents as a challenging emergency not only to the otolaryngologist, but also to the anaesthesiologist.[7] The situation demands a hurried intervention in an often inadequately prepared patient and with not up to the marked instrumentation.

Here, we present a retrospective analysis of cases of various types of foreign body impaction in the upper digestive tract over the past 5 years.

In otolaryngology practice, aspirated or ingested foreign bodies in the upper digestive tract often present as an emergency. The site of impaction and the type of foreign body often determine the urgency of the clinical situation. The clinical presentation of the case has a wide spectrum. From dysphagia, drooling of saliva and retrosternal pain to no complaints. Negative history, normal physical findings and absence of symptoms do not exclude the possibility of foreign body ingestion. ${ }^{[8]}$

A foreign body which has become arrested in the esophagus should be removed as soon as the diagnosis is made since an object once impacted in the esophagus has a small chance of passage, edema from local trauma tends to grip the object more firmly making later manipulation increasingly difficult and perforation of the esophagus is more serious and dangerous than perforation in any other part of the gastrointestinal tract.[9] The dictum of the clinical situation is to remove the impacted foreign body by per oral endoscopy. Not all such impacted foreign body may be removable through per oral endoscopy. At times the situation demands that the foreign body be dislodged into the stomach.

Visualization of the impacted foreign body is difficult at times. Imaging modalities are at times stretched to their limits in trying to locate the impacted foreign body.

Barium studies have a rate of false negative or false positive findings that ranges between 6.5 and $30 \%$ and the risk 
of aspiration in the contrast study.[10] However, Barium Oesophagogram helps in outlining the impacted foreign body. Emergent situation and lack of instrumentation often requires innovative techniques to remove the foreign body.

Impacted foreign body is seen in virtually all age groups, but is most common in the first decade and again in the age group from 6th decade onwards. Varied objects are found impacted like toys and loose dentures. Coin being the most common in children.[11] Meat bone impaction is relatively common in elderly particularly in edentulous patients. In elderly edentulous patients inadequate mastication may be a sufficient explanation for impaction of an abnormally large bolus of food. Also wearing artificial denture, especially the full upper denture can obliterate tactile sensation in the roof of the mouth so that bones and other sharp objects are not detected until they have entered the oropharynx.[12] Sharp objects like pins are not very infrequent, mostly in young patients or those with habit of holding objects in their mouth.

\section{MATERIALS AND METHOD}

A retrospective analysis was done to look at the spectrum of foreign bodies in upper digestive tract, which required admission to the indoor facility of the hospital between February 2009 to February 2015. All patients who presented with history of ingestion of foreign body were included in the review study. All patients of any age and sex who underwent endoscopy and foreign body was recovered were included in the study. Cases with or opharyngeal foreign bodies, who underwent repeat endoscopy or endoscopies done at other center or no foreign body recovered were excluded from the study. We reviewed all patient files with full notations on age, sex, type of foreign body, the anatomical location of impaction and any associated complications. All cases with history of foreign body ingestion were thoroughly evaluated clinically. Pain during swallowing was the major symptom in case of sharp foreign body impaction.

All cases presented with complaints of inability to swallow saliva. Investigations are mainly radiological. X-ray soft tissue neck and chest, AP and lateral views were done in all patients with history of impaction of foreign body in the esophagus. In case of a suspected radiolucent foreign body in the esophagus barium oesophagogram was obtained. CT scan was done only in cases which were still not resolved. At our center, rigid endoscopy of age appropriate size for removal of foreign body has remained the dictum. They were done under general anesthesia. For blunt and non-corroding objects, endoscopy with due preparation was undertaken within 24 hours. In case of corrosive objects and sharp foreign bodies, emergent procedure was undertaken. On recovering the foreign body a second look endoscopy was done to rule out any residual foreign body or any possible erosion or mucosal injury. All post procedure patients were observed for at least twenty four hours to rule out any visceral complications.

\section{RESULTS}

Total no. of cases - 40 .

The youngest patient was 2 years old while the oldest patient was 78 years old. Out of these, 18 were males and 22 were females. The distribution of foreign bodies along the upper digestive tract is summarized in Table no. 4. Most common site of impaction was just below the cricopharynx; 26 patients had foreign body impaction at this level. Chicken or meat bone was the commonest FB followed by coin in the cricopharynx - 15 to 20 cases. Other, relatively uncommon foreign bodies consisted of Safety Pin and a large rusted nail and buttons.
All foreign bodies were radio-opaque except in one case. Removal of FB was done by esophagoscopy under general anesthesia. Flexible oesophagoscopy was done in two cases.

In our series, postoperative recovery in all cases was uneventful except prolonged hospital stay in 2 cases.

SEX

\begin{tabular}{|c|c|c|}
\hline Sex & No. of Patient & Percentage \\
\hline Male & 18 & $45 \%$ \\
\hline Female & 22 & $55 \%$ \\
\hline
\end{tabular}

\section{AGE}

\begin{tabular}{|c|c|c|}
\hline Age GP & No. of Patient & Percentage \\
\hline $0-10$ & 18 & $45 \%$ \\
\hline $11-20$ & 3 & $7.5 \%$ \\
\hline $21-30$ & 3 & $7.5 \%$ \\
\hline $31-40$ & 4 & $10 \%$ \\
\hline $41-50$ & 3 & $7.5 \%$ \\
\hline Above 50 & $\mathbf{9}$ & $\mathbf{2 2 . 5 \%}$ \\
\hline
\end{tabular}

\section{Types of Foreign Bodies}

\begin{tabular}{|c|c|c|}
\hline Types of FB & No. of Patient & Percentage \\
\hline Coin & 15 & $37.5 \%$ \\
\hline $\begin{array}{c}\text { Bone } \\
\text { (Fish/Chicken/Meat) }\end{array}$ & 21 & $52.5 \%$ \\
\hline Open safety pin & 1 & $2.5 \%$ \\
\hline Button & 2 & $5 \%$ \\
\hline Iron nail & 1 & $2.5 \%$ \\
\hline
\end{tabular}

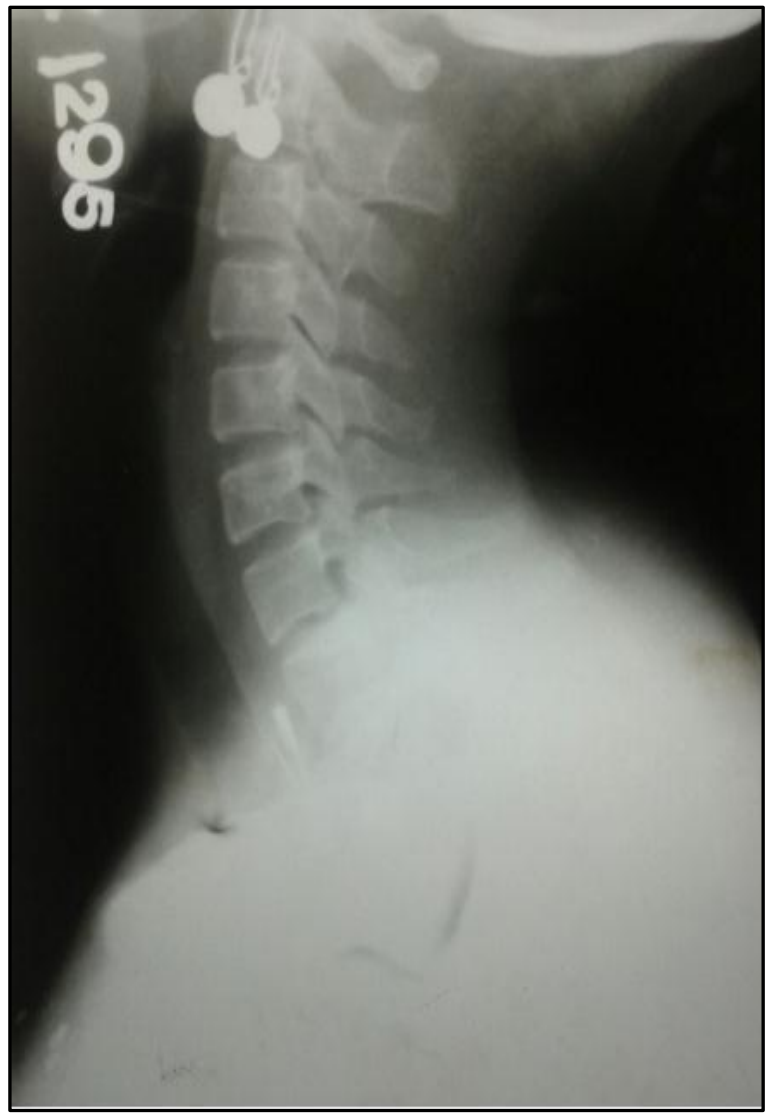

Open Safety Pin 


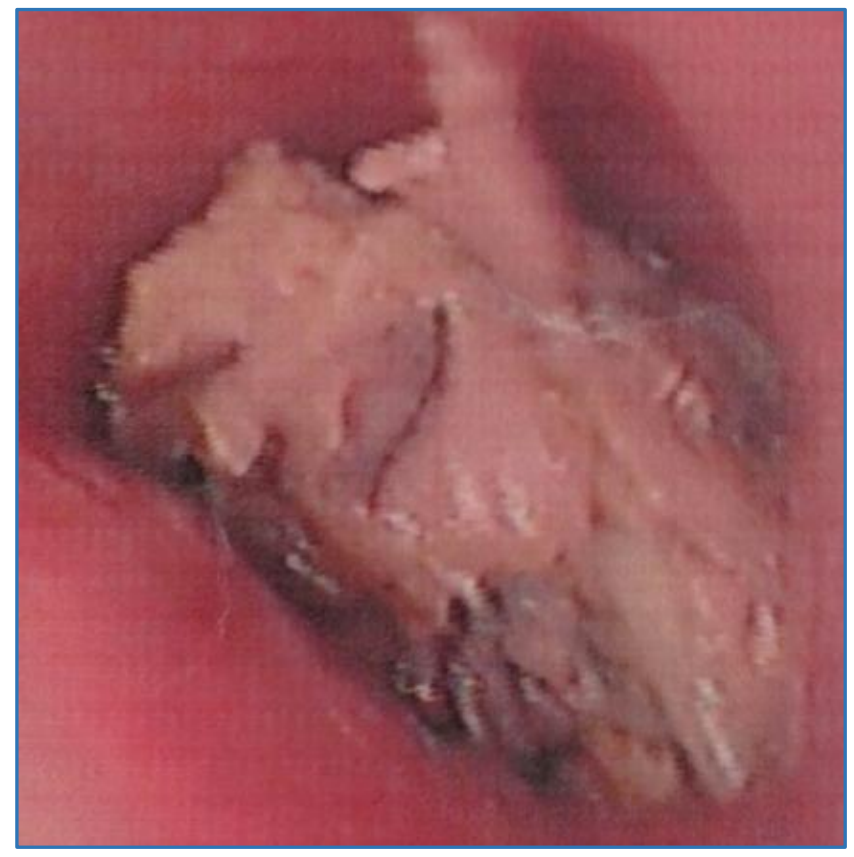

Meat Bolus

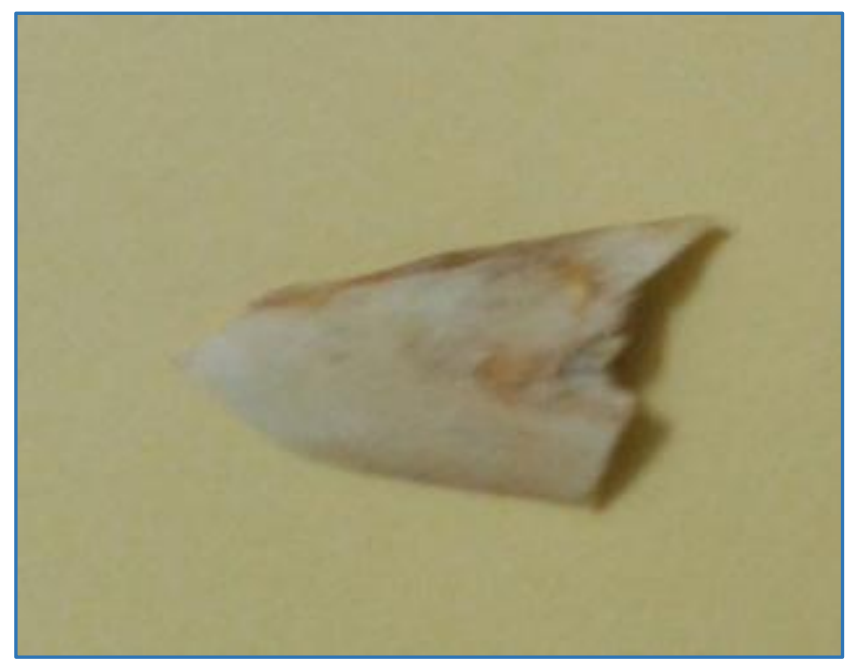

Chicken Bone

\section{Site of Impaction}

\begin{tabular}{|c|c|c|}
\hline Just below Cricopharynx & 26 & $65 \%$ \\
\hline Upper esophagus & 6 & $15 \%$ \\
\hline Mid-esophagus & 2 & $5 \%$ \\
\hline Post cricoid & 6 & $15 \%$ \\
\hline
\end{tabular}

\section{DISCUSSION}

An impacted foreign body in the upper digestive tract can be a potential life-threatening condition if not intervened in time. Commonly the sites of impaction are the natural sites of constriction.[13] Children in their first decade of life have peak incidence. Male and female are almost equally affected.

Aspiration and ingestion of foreign body may be accidental or deliberate in the pediatric age group; it is attributed to their inquisitive nature. Repeated offenders in this age group should undergo psychological evaluation as mental retardation, anxiety disorders and hyperkinetic syndromes can compound the problem.[14-15] In the adult group, majority of the cases are accidental with a small proportion attributed to psychiatric disorders and criminal background, also those with habits of holding foreign objects in their mouth.

Edentulous patients are more prone to accidental ingestion of large and voluminous food bolus leading to impaction or accidental slippage of loose dental prosthesis. Careless ingestion of inadequately masticated food particles during a dining table conversation or under influence of intoxicants also leads to these problems. The presentation may vary over a wide spectrum of symptoms starting with dysphagia, drooling of saliva or even retrosternal pain. In certain cases, there may be no specific symptomatology except a history of suspected foreign body ingestion. Presentation with isolated respiratory symptoms is an enigma that can lead to misdiagnosis.

At the other end of the spectrum are those cases with violent presentation of severe pain, choking and respiratory distress. These can be particularly of serious consequence in the pediatric age group. The spectrum and the prognosis depend on the type of foreign body aspirated and the anatomical site, at which the impaction has occurred.

A detailed history, clinical examination and investigative findings often lead to easy diagnosis. On the contrary an improper history with no objective clinical findings and a negative radiological result can lead to an uncertain situation and may even miss the foreign body. A lateral soft tissue neck radiograph is a cheap readily available investigation tool that is of clinical value in assessing patients with pathology of the upper digestive tract. Prevertebral soft tissue is of variable thickness depending on the level and the age of the patient. In younger children prevertebral tissue can be up to the width of a vertebral body.[16]

In case of a doubtful situation endoscopic clarification should be undertaken. Successful retrieval of impacted foreign body requires a coordinated team work between the endoscopist and the anesthesiologist. A well ventilated and relaxed patient offers the best prospects of a successful retrieval. In recent years either flexible or rigid endoscopes has been the most common approach for removal of impacted esophageal foreign body.

The flexible endoscope is the most preferred method, an over tube conveys all the advantages of a rigid oesophagoscope, flexible endoscopy can most often be performed under local anesthesia in the outpatient setting and the patient recovers rapidly. Thus the expense of hospitalization is avoided.[17-18] In our series it has been our experience that rigid endoscopy offers an easier and better opportunity to retrieve foreign bodies. This may well be attributed to our lack of experience with flexible fiberoptic scopes in retrieval of impacted foreign bodies.

Foreign bodies ingested have been found to be lodged usually just below the cricopharynx probably because of the strong peristaltic movement of the constrictors and the relatively weak propelling power of the oesophageal muscles. An impacted foreign body must be removed at the earliest to prevent complications like progressive inflammation and edema of the overlying mucosa, adding to the already impacted foreign body difficult to locate, making manipulation more risky.

\section{CONCLUSION}

Foreign body in the upper digestive tract can have varied presentation.

In the pediatric age group a high degree of suspicion is required.

Once confirmed, the foreign body should be removed at the earliest opportunity to prevent further complication.

In our series, incidence was found to be almost equal between male and females. 
In our series, we found the use of a rigid scope more easy and helpful for removal of foreign bodies.

Parents should be counseled to be aware of the possible grave complications that can occur in case of a neglected foreign body.

\section{REFERENCES}

1. Biswas B, Datta R. Retained Oesophageal Foreign Bodies Report of three cases. Indian J Otolaryngol Head Neck Surg, 1999 Aug;vol 51 (Suppl 1);15-18.

2. Dov Weissberg MD, Yael Rafaely MD. Foreign bodies in the oesophagus. Ann Thoracic Surg 2007;84:1854-7.

3. Shiva Kumar AM, Ashok S Naik, Prashanth KB, et al. Foreign bodies in the upper digestive tract. Indian J otolaryngol head neck surg. Jan 2006 Vol 58 issue 1 pp 63-68.

4. Yang CY. The management of ingested foreign bodies in the upper digestive tract; a retrospective study of 49 cases. Singapore Med J 1991 Oct;32(5):312-5.

5. Kalliopi Athanassiadi, et al. Management of esophageal foreign bodies: a retrospective study of 400 cases. European Journal of Cardiothoracic Surgery. - vol 21, issue - 4 Pp $653-656$.

6. Sadan N, Ras A, Wolach B. Impact of community educational programme on foreign body aspiration in Israel. Eur J Pediatrics 1995;154:859-62.

7. Muranjan $\mathrm{M}$, Bavdekar $\mathrm{S}$, Batra $\mathrm{H}$, et al. Unusual aerodigestive tract foreign bodies; tribulations and tragedies. Int J Pediatric Otolaryngology 2005 Sep;69(9):1269--74

8. Sule Yalcin, et al. Foreign body ingestion in children; an analysis of pediatric surgical practice. Pediatric Surgery International. Aug 2007; vol 23 issue 8: pp 755-761.
9. Nandi P, Ong GB. Foreign body in the oesophagus. Review of 2394 cases. Br J of Surg 1978;65:5-9.

10. Moralles-Angulo C, Rodriguez Iglesias J, Mazon Gutierrez $\mathrm{A}$, et al. Foreign bodies in the oesophagus. Acta Otorhinolaryngol Esp 1998 Nov-Dec;49(8):644-6.

11. Moralles-Angulo C, Rodriguez Iglesias J, Mazon Gutierrez $\mathrm{A}$, et al. Foreign bodies in the oesophagus. Acta Otorhinolaryngol Esp 1998 Nov-Dec;49(8):644-6.

12. Bakara A, Bikhazi G. Oesophageal foreign bodies. BM] 1975;1:561-3.

13. Emara MH, Darwiesh EM, Refaey MM, et al. Endoscopic removal of foreign bodies from the upper gastrointestinal tract; 5 year's experience. Clin Exp Gastroenterol 2014 Jul 16;7:249-53.

14. Saha S, Mishra S, Chakraborty DD, et al. Occult foreign bodies in the aerodigestive tract in infancy. Indian Journal of Otolaryngology and Head and Neck Surgery. 2008 Mar;60(1):37-40. E pub 2008 Apr 3.

15. Murty P, Ingle VS, Ramakrishna S, et al. Foreign bodies in the upper aerodigestive tract. J Sci Res Med Sci 2001 Oct;3(2):117-20.

16. Jagdeep Singh Virk, Jingyin Pang, et al. Analysing lateral soft tissue neck radiographs. June 2012 , vol 19, issue 3, pp 255-260.

17. Brady PG. Esophageal foreign bodies. Gastroenterol Clin North Am 1991 Dec;20(4):691-701.

18. Xiu-eYan, Li-ya Zhou, et al. Therapeutic effect of esophageal foreign body extraction management: Flexible versus rigid endoscopy in 216 adults of Beijing, Med Sci Monit 2014;20:2054-2060. 